\title{
Über Eponyme und das Verteilen von Ehre in der Medizin
}

\author{
Eberhard Wolff \\ Prof. Dr. rer. soc., Redaktor Kultur, Geschichte, Gesellschaft
}

Eine der Leidenschaften der Medizin besteht bekanntlich darin, Ehre zu verteilen. Zum Beispiel erfreuen sich Preise in diesem Metier einer ausgesprochenen Beliebtheit. Auf den ersten Blick erscheint das ganz einfach: Eine Fachgesellschaft bedenkt eine verdiente Person des Fachs mit Ehre, indem sie ihr einen wohlklingenden Preis mit einem mehr oder weniger hohen Preisgeld verleiht.

Ganz so einfach ist das aber nicht. In der Regel wird nämlich gleich zwei Mal Ehre ausgeteilt. Einerseits natürlich an die Preistragenden, andererseits sind die Preise oft mit den Namen älterer oder bereits verstorbener Kollegen (seltener: -Innen) versehen, die damit ebenfalls mit Ehre bedacht werden. Haben diese dann bereits klingende Namen, reflektieren sie wiederum ihr Ansehen auf die Preisvergebenden zurück. Und manchmal soll ja auch ein illustrer Preisträger das Image eines etwas unscheinbaren Preises aufpoliert haben. So eine Preisvergabe ist also ein richtiger «Ehrenhandel».

Dieser Handel ist aber noch komplizierter. Für den französischen Kultursoziologen Pierre Bourdieu sind Ehre und Ansehen ein «symbolisches Kapital». Und das kann in soziales oder ökonomisches Kapital umgemünzt werden. Bleiben wir beim Beispiel des Preises: Zumindest für die Lebenden sollte sich die Ehre des Preisgewinns auch in gesellschaftlicher Anerkennung, beruflichem Aufstieg und damit letzten Endes Einfluss und Einkommen niederschlagen.

\section{Wenn Ehre verteilt wird, laufen offen- sichtlich komplizierte Prozesse ab}

Eine andere Subspezies der Ehrverteilung ist in der Medizin ganz besonders endemisch: die Eponyme. Krankheiten, Syndrome, Gerätschaften, Methoden/ Verfahren oder Institutionen werden nach Menschen benannt, die sie (mehr oder weniger bzw. angeblich) erstbeschrieben, entdeckt, entwickelt, gegründet, geleitet oder gefördert haben. Die grosse Zeit der Eponyme war etwa in den letzten zwei Jahrhunderten. Es war auch die grosse Zeit der pathetischen Ehrungsdenkmäler.
Heute werden komplexe medizinische Theorien, Verfahren und Instrumente viel öfter von grossen Teams entwickelt. Eponyme werden jetzt zunehmend durch handliche Abkürzungen, die sogenannten Akronyme, verdrängt. Trotzdem sind Eponyme in der Medizin bis heute alltäglich.

Bereits die Wege, auf denen Eponym-Ehre entsteht, sind kompliziert. Manchmal beschliessen Fachgesellschaften. Manchmal können medizinische Schulen einen Namen in der Praxis durchsetzen. Manchmal arbeiten die Namensgeber selber daran. Mediziner haben in den letzten Jahrhunderten immer wieder spezielle Geburtszangen, chirurgische Klemmen oder andere Instrumente entwickelt, die oft nur ein bisschen anders als die bekannten Varianten konstruiert waren - aber anders genug, um den eigenen Namen zu tragen. Um die Ehre der Eponyme herrscht ein ordentliches Machtgerangel.

Ganz abgesehen davon, dass die Ehre der Eponyme alles andere als gerecht verteilt wird. Es ist keine Ausnahme, dass ein Syndrom von jemand anderem schon früher beschrieben oder eine Methode von mehr als nur dem Geehrten entwickelt wurde.

Eponyme sollen die Ehre eines unsterblichen Angedenkens verleihen. In der Praxis ist das alles aber auch nicht so einfach. Erinnern Sie sich an Finding Nemo und die grandiose Szene in der Zahnarztpraxis? Wie Profis kommentieren die Fische vom Aquarium aus eine Wurzelbehandlung mit dem Gates-Bohrer («den benützt er öfter in letzter Zeit») und der Schilder-Methode. Dann streiten sie, ob der Zahnarzt gerade eine Hedström- oder eine Kerr-Feile ansetzt. Der Nachruhm des schwedischen Zahnarztes Erik Gustav Hedström (1869-1948) hielt sich trotz des Eponyms in Grenzen. Heute muss man sich bis in die schwedische Wikipedia durchgoogeln, um wenigstens ein paar Zeilen über ihn zu finden. Bald wurde der Name seines Instruments zur «H-Feile» verkürzt - und die «Kerr-» zur «K-Feile». Im englischen Filmoriginal spricht der bunte KönigsFeenbarsch «Gurgle» (in der deutschen Version «Sushi») gleich von einer «K-Flex». Und wer dieser «Dr. Gates» mit seinem Bohrer war, ist der Fachwelt bis heute offensichtlich unklar. Im englischen Filmoriginal ist es übri- 
gens ein «Gates-Glidden»-Bohrer, aber über den Dr. Glidden weiss man auch nicht viel mehr [1]. Einzig der hinter der Schilder-Methode (den Wurzelkanal zu füllen) stehende Herbert Schilder (1928-2006) ist heute ein bekannterer Name. Schilder war über seine Methode hinaus eine illustre Figur der Zahnmedizin und lebte noch, als der Nemo-Film entstand. Ganz so unsterblich macht das Eponym für sich also doch nicht.

Noch problematischer ist die Ehrungsform der Eponyme, wenn die dadurch geehrten Forscher offensichtlich selbst nicht so ehrenhaft waren. Die nördlichen Nachbarn können ein Lied davon singen. Eponyme sind zum Teil nach Forschenden benannt, die im Nationalsozialismus "ethisch fragwürdige Forschung» betrieben haben. Der Fachverband der deutschsprachigen Medizingeschichte richtete kürzlich in Mainz ein Kolloquium dazu aus [2]. Als älteres, prominentes Beispiel unter vielen wird immer wieder das «ReiterSyndrom» angeführt. Der Bakteriologe und hohe NaziFunktionär bzw. -Propagandist Hans Reiter (1881-1969) wurde für medizinische Experimente im KZ Buchenwald als Kriegsverbrecher verurteilt. Heute wird das Syndrom deshalb mehrheitlich als «Reaktive Arthritis» bezeichnet. Aktuell gibt es die Diskussion um das "Asperger»-Syndrom, nachdem gezeigt wurde, dass Hans Asperger (1906-1980) in Wien aktiv mit dem Programm der Kinder-Euthanasie kooperiert hat [3].

Was tun? Auch hier gibt es keine einfache Lösung. Ganz auf Eponyme zu verzichten wäre in der Praxis kaum umzusetzen. Die Forderung nach Umbenennung einzelner Täter-Eponyme ist im Kern nur allzu nachvollziehbar. Die Probleme zeigen sich dann aber im Detail. Wo werden die Grenzen gezogen? Wer entscheidet darüber?

Ganz ähnliche Probleme werden im Umgang mit historischen Denkmälern verhandelt. Mit den Eponymen haben sie nicht nur gemein, dass sie Beehrungs-Techniken, sondern (so Matthis Krischel, Düsseldorf, auf der genannten Tagung) auch Orte der Erinnerungskultur sind. In den USA herrscht in letzter Zeit ein regelrechter Kampf um Denkmäler für Südstaatengeneräle, die für die Sklavenhaltung Krieg führten. Kann man die öffentliche Ehrung solcher Leute heute noch vertreten? Ist ein Abbau nicht einfach eine Geschichtsentsorgung? Sind die Denkmäler Chancen für eine Auseinandersetzung mit der Geschichte? Haben die Südstaatler ein Anrecht auf ihre eigene Geschichte? Und überhaupt: Werden die Denkmäler heute noch so wahrgenommen, wie sie gedacht waren? In abgeschwächter, aber ähnlicher Weise gilt dies ebenso für die zurückhaltenderen Tell-, Winkelried-, GeneralGuisan- und Rütlischwur-Denkmäler in der Schweiz. Und damit kommen wir wieder zu unserem eigentlichen Thema zurück. Die in Denkmälern genauso wie in Eponymen oder Preisen auskristallisierte Ehre hat offenbar eine Halbwertszeit. Die Ehre von damals ist nicht immer die Ehre von heute. Oft schrumpft ihre Macht mit der Zeit. Das hat der oben erwähnte Pierre Bourdieu formuliert. Das symbolische Kapital des Ansehens kann nur dann als Machtmittel wirken, wenn es allgemein anerkannt ist.

Ehre ist eine beliebte, aber unsichere Währung und ein schlüpfriger Boden, auf dem man schnell ausrutschen kann. Das sollte man sich klarmachen, bevor man mit dem Verteilen und Annehmen von Ehre beginnt. Vielleicht hat aus diesem Grund der Zürcher Naturarzt Max Bircher sein Müesli immer uneponymisch "Apfeldiätspeise» genannt. Und vielleicht hatte die im letzten Jahr verstorbene Pädiaterin Ingeborg (Syllm-)Rapoport etwas Ähnliches im Sinn. Als die Ärztin am Hamburger Israelitischen Kran-

kenhaus 1937 ihre Dissertation einreichte, wurde dies der Jüdin im Nazi-Deutschland verweigert. Nach dem Krieg machte sie in der DDR Karriere und blieb auch nach dem Mauerfall noch überzeugte Kommunistin. Zu ihrem 100. Geburtstag bot die Universität Hamburg ihr als Zeichen der Wiedergutmachung den Ehrendoktortitel an. Rapoport aber zog der Ehrenbezeugung eine richtige Doktorprüfung (mit 77-jähriger Verspätung) vor und wurde im Alter von 102 Jahren regulär promoviert. Natürlich war dies das stärkere Signal gegen das Unrecht. Vielleicht hatte sie aber auch befürchtet, ihre aktive Rolle im SED-Staat hätte bei einem Ehrendoktor $\mathrm{zu}$ unangenehmen Ehrenhändeln führen können.

\section{Literatur}

1 Guttman JL, Formantin K. The Gates-Glidden bur: the search continues. J Hist Dent. 2015;63(1):22-8.

2 Kolloquium «Aktuelle Debatten um die Verwendung von medizinischen Eponymen: Die Position der Medizingeschichte» des Fachverbands Medizingeschichte in Mainz vom 29. Juni 2018. Das Programm unter https://www.fachverband-medizingeschichte.de/ kolloquien. Einige Anregungen und Informationen für diesen Artikel habe ich diesem Kolloquium entnommen. Ich danke den Beteiligten.

3 Czech H. Hans Asperger, National Socialism, and «race hygiene» in Nazi-era Vienna. Molecular Autism. 2018;9:29.

Bildnachweis

(c) Somesun | Dreamstime.com 